\title{
STREAMING MOTIONS IN THE LOCAL UNIVERSE
}

\author{
Ofer Lahav \\ Institute of Astronomy \\ Madingley Road \\ Cambridge, CB3 0HA. \\ England
}

\begin{abstract}
Deviations from the Hubble flow directly probe of the underlying total mass distribution, assuming the gravitational instability picture. We discuss the origin of motion of the Local Group with respect to the Cosmic Background Radiation and review the peculiar velocity field deduced from distances to hundreds of elliptical and spiral galaxies, including new results for the Shapley Supercluster. Bulk-flow solutions which are free of Malmquistbias are presented, indicating coherence length larger than that expected from the optical and IRAS dipoles or from Cold Dark Matter models.
\end{abstract}

\section{The Origin of Motion of the Local Group}

The best known peculiar velocity is that of the sun as deduced from the dipole anisotropy of the Cosmic Background Radiation (CBR). This corresponds to a Local Group velocity of about $600 \mathrm{~km} / \mathrm{sec}$ with respect to the CBR towards $l \approx 268^{\circ} ; b \approx 27^{\circ}$.

The origin of the motion can be explored by estimating the acceleration vector ('dipole') due to catalogued galaxies using just their fluxes (since both gravity and flux fall as the square of distance) or including redshift information. In linear theory the acceleration is parallel to the peculiar velocity $\mathbf{v}$

$$
\mathbf{v} \propto \frac{f\left(\Omega_{0}\right)}{b} \int\left(\frac{\delta \rho}{\rho}\right) \frac{\hat{\mathbf{r}}}{r^{2}} d^{3} r
$$

where $f\left(\Omega_{0}\right) \approx \Omega_{0}^{0.6}$ (Peebles 1980$)$. When generalized to include the cosmological constant $\lambda_{0} \equiv \Lambda /\left(3 H_{0}^{2}\right)$, there is very little change, $f\left(\Omega_{0}, \lambda_{0}\right) \approx \Omega_{0}^{0.6}+\frac{1}{70} \lambda_{0}\left(1+\frac{1}{2} \Omega_{0}\right)$ (Lahav et al. 1991). The 'bias parameter' $b$ is commonly introduced to take into account that galaxies of particular type may not be perfect tracers of the mass fluctuations $\frac{\delta \rho}{\rho}$.

Various recent estimates of light dipoles all lie within $20^{\circ}$ of the $\mathcal{C} B R$ dipole. Figure 1 shows the growth of the amplitudes of two IRAS dipoles and an optical dipole. We see that the 3 curves level off at about $4000 \mathrm{~km} / \mathrm{sec}$, suggesting that about $80 \%$ of the dipole is generated within a radius of $4000 \mathrm{~km} / \mathrm{sec}$ (although the QDOT dipole may suggest contribution from larger distances). Given a sample of a finite depth, it is possible that there is a contribution to the acceleration due to matter outside the sample. But for example the Shapley Supercluster which is at the same direction of Centaurus/Great-Attractor, but at 
a distance of $14000 \mathrm{~km} / \mathrm{sec}$, contributes no more than $15 \%$ (of the optical dipole) to the motion of the Local Group (Raychaudhury 1989).

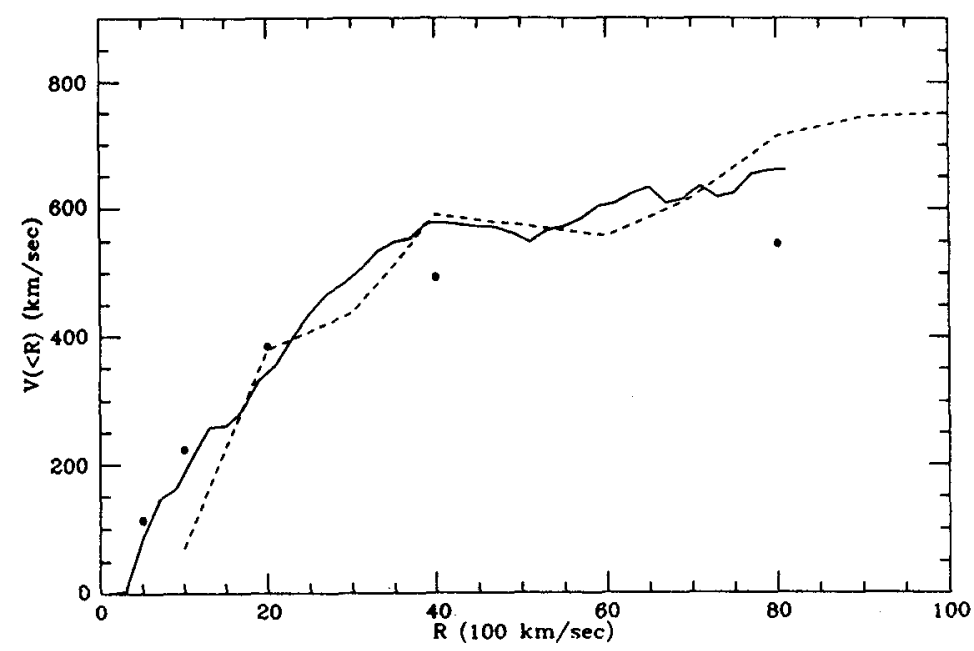

Figure 1. The growth of light dipoles as a function of the radius of a sphere centred at the Local Group. The solid line shows the dipole from a 2 Jy IRAS redshift survey (Strauss \& Davis 1988), the dashed line from the QDOT 0.6 Jy IRAS redshift survey (Rowan-Robinson et al. 1990), and the dots are from an optical dipole deduced from the UGC and ESO catalogues (Lynden-Bell, Lahav \& Burstein 1989). The curves are normalized to agree at about $R=2000 \mathrm{~km} / \mathrm{sec}$.

It is worth emphasizing that the so-called 'bias parameter' appears in different ways in different measurements of the galaxy and matter density fields. When discussed in terms of the variance of a density field in (say) spheres of radius of $800 \mathrm{~km} / \mathrm{sec}$, the relation between the fuctuations in galaxy density and the matter density is commonly written as $\left\langle\left(\frac{\delta n}{n}\right)^{2}\right\rangle_{8}^{1 / 2}=b\left\langle\left(\frac{\delta \rho}{\rho}\right)^{2}\right\rangle_{8}^{1 / 2}$. In these spheres the rms for optical galaxies is roughly unity, but for IRAS galaxies one gets only 0.7 , so $b_{O} / b_{I} \approx 1.4$. However, when the dipole statistic is discussed, a different moment of the density field is considered (eq. 1) over scale of about $10000 \mathrm{~km} / \mathrm{sec}$. The two estimates for $b$ need not agree in principle (unless at every point in space $\left.\frac{\delta n}{n}=b \frac{\delta \rho}{\rho}\right)$. In practice, we find from the optical dipole $\Omega_{0}^{0.6} / b_{O} \approx 0.4$, while the IRAS one gives $\Omega_{0}^{0.6} / b_{I} \approx 0.8-1.0$. So the ratio of the bias parameters is $b_{O} / b_{I} \approx 2$, not far from that deduced from the variance on much smaller scales.

\section{Line-of-Sight Peculiar Velocities}

It is possible to deduce the line-of-sight peculiar velocity of a galaxy by measuring its redshift and its distance, i.e. $V_{p}=c z-H_{0} r$. This way of mapping the peculiar velocity field was pioneered by Rubin and collaborators and in recent years several groups (e.g. Aaronson et 
al. 1986, Lynden-Bell et al. 1988) have used various distance indicators like the infra-red Tully-Fisher (luminosity-line width) relation for spirals and the diameter-velocity dispersion $\left(D_{n}-\sigma\right)$ relation for ellipticals. The distance indicators show no dependence on environment, e.g. as a function of the distance from the centre of Coma (Lucey et al. 1991).

Assuming that the peculiar velocities are 'real', the 7 Samurai argued that Centaurus is moving, and hence proposed a massive object (the "Great Attractor") behind it, at about $4300 \mathrm{~km} / \mathrm{sec}$. Dressler \& Faber (1990) claimed an evidence for a 'backside infall' into the 'Great Attractor'. However, Mathewson et al. (1991) recently argued that the flow continues, at least out to $6000 \mathrm{~km} / \mathrm{sec}$. How far does the flow continue? According to one $D_{n}-\sigma$ survey (Lucey, Lahav, Lynden-Bell, Terlevich, Melnick and Infante; in preparation) the cluster Shapley 8, a member of the Shapley Supercluster at $14000 \mathrm{~km} / \mathrm{sec}$, is consistent with bcing at rest with respect to the $C B R, V_{p}=-300 \pm_{600}^{1300} \mathrm{~km} / \mathrm{sec}$, although the error bars are fairly large, as expected at such large distances.

There is clearly a need for new techniques to deduce peculiar velocities such as the kinematic SZ effect (Sunyaev \& Zeldovich 1980; Rephaeli \& Lahav 1991) or surface brightncss fluctuations analysis (Tonry et al. 1989).

\section{Malmquist Bias Revisited}

The deduction of distances suffers a bias since the distance-indicators are drawn from a distribution (either cosmic or due to measurement errors). This kind of problems has been discussed by Eddington and Malmquist already in the 1920's in relation with star counts. For recent discussion see e.g. Sandage, Tammann \& Yahil (1979), Aaronson et al. (1986), Lynden-Bell et al. (1988), Tully (1988) and Faber \& Burstein (1988). The distance to an elliptical galaxy can be estimated as $r \propto \sigma^{1.2} / D_{n}$, where $D_{n}$ is an angular diameter. If the distribution of the galaxies is uniform there is a higher probability for finding a galaxy at larger distances (due to a larger volume). Similarly, there are biases if a galaxy is likely to reside in a dense region, or due to observational selection effects. The 7 Samurai (Lynden-Bell et al. 1988) have corrected each observed distance of an individual galaxy by multiplying it by about 1.15, assuming that elliptical galaxies are distributed uniformly (clearly an unrealistic assumption). Here we show, from work in progress with D. Lynden-Bell, another approach to the problem, based on a procedure used by Schechter (1980). The key idea is to treat the distance-independent parameter ( $\sigma$ in our case) as the independent variable, and to write the conditional probability for the velocity dispersion given other observables and parameters, $P\left(\sigma \mid \hat{\mathbf{r}}, c z, D_{n}\right.$, flow model, $\left.\ldots\right)$, which we take to be a Gaussian in $\log \sigma$. Note that the predicted $\sigma$ depends on a flow model (with free parameters) and on the 'field velocity dispersion' $\sigma_{f}$. For more details see Lynden-Bell (1991). One can then write a Likelihood function for all the galaxies observed and solve for the parameters of interest. In this method the selection effects and the density field of the galaxies can be neglected, and it provides good solution for large distances. We have used D. Burstein's compilation of $D_{n}-\sigma$ for 544 individual ellipticals. For galaxies in rich clusters we assigned the cluster velocity dispersion, while for the 'field' galaxies $\sigma_{f}$ was left as a free parameter.

For a pure bulk flow model of the ellipticals sample with respect to the CBR (assuming $\sigma \propto D_{n}^{0.75}$ based on Coma) we found $\sigma_{f} \approx 350 \mathrm{~km} / \mathrm{sec}$ and a streaming motion of $U(l, b)=$ $501(297,15) \mathrm{km} / \mathrm{sec}$ (the error in each component is less than $100 \mathrm{~km} / \mathrm{sec}$ ). For comparison, the 7 Samurai found using their method 521(307,9) and Lilje et al. (1986) found from 
spirals 502(288,9). Excluding the Centaurus region from our analysis makes little difference, indicating that the bulk flow is a global pattern, not due just to Centaurus.

Next we allowed for radial dependence. For a Top-Hat we get $U(l, b)=542(302,18)$ $\mathrm{km} / \mathrm{sec}$, with a radius of $5400 \mathrm{~km} / \mathrm{sec}$, indicating a large coherence length. As more general and objective family of models, we expanded the density field in spherical harmonics (cf. Regos and Szalay 1989; Scharf et al. 1991). Then using Poisson's equation and assuming that the velocity is a gradient of the potential, we can write the predicted line-of-sight velocity as $U_{l o s}(\mathbf{r})=\sum_{l} \sum_{m} u_{l m}(r) Y_{l m}(\theta, \phi)$, with particular set of radial functions $u_{l m}(r)$. Our preliminary results indicate that the data show no sign of 'backside infall', and that the coherence length is larger than that expected from the optical and IRAS dipoles.

\section{Cosmological Implications}

We discussed the deduction of of $\Omega_{0}^{0.6} / b$ from the dipole at our position. A comparison of the velocity field and IRAS galaxies at many other points (Bertschinger et al. 1990, Yahil 1990 , Kaiser et al. 1991 ) gives $\Omega_{0}^{0.6} / b \approx 1.0 \pm 0.3$, somewhat higher but still consistent with the estimate from the local IRAS dipole. Another approach is to confront the observations directly with the velocities expected in theoretical models for the primordial density fluctuations. The most commonly used and robust statistic is the bulk flows statistic (e.g. Kaiser 1988). Other statistics include the velocity correlation function (Gorski et al. 1989; Groth et al. 1989), the 'Mach number' (Ostriker \& Suto 1989), and the dipole's misalignment angle (Juszkiewicz et al. 1990; Lahav et al. 1990).

In the bulk flow statistic the measurement of the flow on a given scale $R_{*}$ is compared with the rms expected in a hypothetical universe specified by a given power-spectrum $P(k)$ and a 'window function' $W\left(R_{*} k\right)$ which describes the observational set-up:

$$
\left\langle V_{p}^{2}\left(R_{*}\right)\right\rangle=H_{0}^{2} \Omega_{0}^{1.2} \frac{1}{(2 \pi)^{3}} \int d^{3} k \frac{1}{k^{2}} P(k) W\left(k R_{*}\right) .
$$

The freedom here is the normalization of the power spectrum which can be specified by the rms fluctuation $\sigma_{8}$ on $800 \mathrm{~km} / \mathrm{sec}$ sphere.

A general Cold Dark Matter spectrum depends on $\Omega_{0} h$; The smaller $\Omega_{0} h$, the more powcr there is at large scale. Indeed, Efstathiou et al. (1990) fitted their APM angular two-point correlation function by a CDM model with $\left(\Omega_{0}=0.2 ; h=1\right)$. However, when velocities are considered $\Omega_{0}$ also controls the strength of gravity by the $\Omega_{0}^{0.6}$ factor. In Figure 2 we show the predictions of bulk motions for this model, for a Gaussian filter, $W\left(k R_{*}\right)=\exp \left(-k^{2} R_{*}^{2}\right)$. For comparison we also show the standard CDM model $\left(\Omega_{0}=1.0, h=0.5\right)$. Although the value of $R_{*}$ is controversial (Kaiser 1988; Kaiser \& Lahav 1989), we see that for unbiascd models the low-density CDM model gives peculiar velocities lower than the standard model for any $R_{*}$. As indicated in the figure for the case $\left(\Omega_{0}=0.2, h=0.5\right)$, more power can be provided by decreasing the Hubble constant. If the large streaming motions reportcd by Willick (1990) and Mathewson et al. (1991) are real, 'conventional' models do not have enough power at large scales to match the observations. 


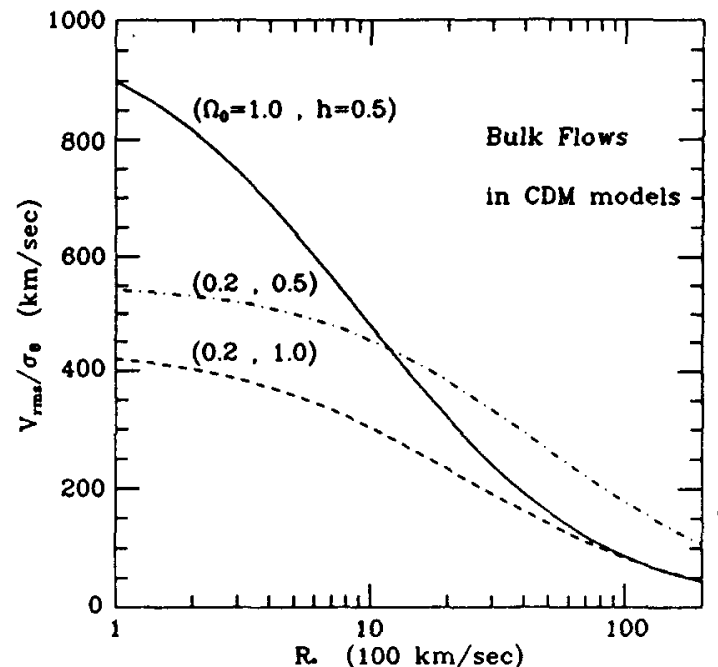

Figure 2. Predicted rms flows for a family of Cold Dark Matter models characterized by $\Omega_{0}$ and $H_{0}=100 \mathrm{hm} / \mathrm{sec} / \mathrm{Mpc}$.

\section{References}

A arouson, M, et al. , 1986. Ap. J., 302, 536.

Bertschinger, E. \& Dekel, A., Faber, S.M., Dressler, A., \& Burstein, D., 1990. Ap. J., 364 370

Dressler, A. \& Faber, S.M., 1990. Ap. J., 354, L45.

Efstathiou, G., Sutherland, W.J. \& Maddox, S.J., 1990. Nature, 348, 705.

Faber, S.M. \& Burstein, D., 1988. in Large Scale Motions in the Universe, eds. G. V. Coyne \& V.C. Rubin, Princeton University Press, Princeton.

Gorski, K. et al. 1989. Ap. J, 344, 1.

Groth, E.J., Juszkiewicz, R. \& Ostriker, J.P., 1989. Ap. J., \$48, 558

Juszkiewicz, R., Vittorio, N. \& Wyse, R., 1990. Ap. J., 348, 408.

Kaiser, N., 1988. M.N.R.A.S., 2\$1, 149.

Kaiser, N. \& Lahav, O., 1989. M.N.R.A.S, 287, 129.

Kaiser, N. et al. , 1991. M.N.R.A.S., $252,1$.

Lahav, O., Kaiser, N. Hoffman, Y., 1990. Ap. J., 352, 448.

Lahav, O., Lilje, P.B., Primack, J.R., \& Rees, M.J., 1991. M.N.R.A.S., 251, 128.

Lilje, P.B., Yahil, A. \& Jones, B.J.T., 1986. Ap. J., s07, 91.

Lucey, J.R., Guzman, R., Carter, D. \& Terlevich, R. J. , 1991. preprint.

Lynden-Bell, D., Faber, S.M., Burstein, D., Davies, R.L., Dressler, A., Terlevich, R.J. \& Wegner, G., 1988. Ap. J., 326, 19.

Lynden-Bell, D., Lahav, O. \& Burstein, D., 1989. M.N.R.A.S., 241, 325

Lynden-Bell, D., 1991. In "Statistical Challenges in Modern Astronomy", eds. G.J. Babu \& E.D. Feigelson.

Mathewson, D.S., Ford, V.L. \& Buchhorn, M., 1991. preprint.

Ostriker, J.P. \& Suto, S., 1990. Ap. J., 348, 378.

Peebles, P.J.E., 1980. The Lange Scale Structure of The Universe, Princeton University Press, Princeton.

Raychandhury, S., 1989. Nature, 342, 251.

Regos, E. \& Szalay, A.S., 1989. Ap. J., 343, 627.

Rephaeli, Y. \& Lahav, O., 1991. Ap. J., $372,21$.

Rowan-Robinson et al. , 1990. M.N.R.A.S. 247, 1.

Sandage, A., Tammann, G.A. \& Yahil, A., 1979. Ap. J., 232, 352.

Scharf, C., Hofman, Y., Lahav, O. \& Lynden-BeII, D., 1991. preprint.

Schechter, P., 1980. Astr. J., 85, 801.

Strauss, M.A. \& Davis, M., 1988. in Lange Scale Motions in the Universe, eds. G. V. Coyne \& V.C. Rubin, Princeton Unjversity Press, Princeton.

Sunyaev, R.A. \& Zeldovich, Ya.B., 1980. M.N.R.A.S., 180, 413

Tonry, J.L., Ajhar, E.A. \& Luppino, G.A., 1989. Ap. J., 346, L57.

Tully, R.B., 1988. Nature, 334, 209.

Yahil, A. 1990. in Proceedings of the Moriond conference, March 1990.

Willick, J.A. 1990. Ap. J, 351, L5. 